\title{
Remarks about diffusion mediated transport: thinking about motion in small systems
}

\author{
Stuart Hastings \\ Department of Mathematics \\ University of Pittsburgh, \\ Pittsburgh, PA 15260, USA, \\ E-mail: sph+@pitt.edu \\ David Kinderlehrer * \\ Center for Nonlinear Analysis and \\ Department of Mathematical Sciences \\ Carnegie Mellon University, \\ Pittsburgh, PA 15213, USA, \\ E-mail: davidk@andrew.cmu.edu
}

January 27,2004

Dedicated to the memory of Guido Stampacchia

\begin{abstract}
We describe a dissipation principle/variational principle which may be useful in modeling motion in small viscous systems and provide brief illustrations to brownian motor or molecular rachet situations which are found in intracellular transport. Monge-Kantorovich mass transport and Wasserstein metric play an interesting role in these developments. Some properties of the system that ensure the presence of transport are discussed.
\end{abstract}

\section{Introduction}

Here we describe a dissipation principle that describes transport in a typical molecular motor system, like conventional kinesin, [20], [22]. As background to this application, we recount that intracellular transport in eukarya is attributed to motor proteins that transduce chemical energy into directed mechanical motion. Muscle mysosin has been known since the mid-nineteenth century and its role in muscle contraction demonstrated by A.F. Huxley and H.E. Huxley in the 1950's.

*Partially supported by the National Science Foundation Grants DMS 0072194 and DMS 0305794. 
Kinesins and their role in intracellular transport were discovered around 1985. These nanoscale motors tow organelles and other cargo on microtubules. They function in a highly viscous setting with overdamped dynamics; the Reynolds' number is about $5 \times 10^{-2}$. The dissipation principle begins a chain of events. It suggests, in a natural way, a variational principle and an implicit scheme in the sense of Otto [14], [15] and Jordan, Kinderlehrer and Otto [9]. This determines, in turn, a system of equations analogous to that proposed by Adjari and Prost [1] or Peskin, Ermentrout, and Oster [18]. Viewed as an ensemble, this system occupies configurations that are distant from conventional notions of equilibrium. This means that to understand the stability properties of the process we must discover an appropriate environment for its kinetics. The novelty in our development is that the dynamical process is set in a weak topology as described by a KantorovichWasserstein metric. This owes in part to a result of Brenier and Benamou, [3]. It illustrates the feasibility of mesoscale modeling for these systems.

The flashing rachet, a different type of Brownian motor, was discussed in [10]. One explanation of this was given in [2] and it has been suggested as a description of processivity in the KIF-1A family of kinesins, [12], [13]. There is a discussion in [6] as well as the Parrondo Paradox, a coin toss game somethimes thought to mimic molecular motor behavior, in [7].

With a thermodynamically consistent system of differential equations in hand, we inquire of conditions that ensure transport. In the example we describe, a model for conventional kinesin, diffusion and conformational change collaborate with transport in periodic potentials. This model is highly over simplified. Asymmetry of the potentials within their period intervals is critical for transport, and a particular such condition based on this property is explained.

This is a description of joint work with Michal Kowalczyk, Michel Chipot, and Jean Dolbeault, to whom we are grateful for their collaboration.

\section{A variational principle}

Consider an ensemble of statistically homogeneous non-interacting particles in a highly viscous medium, thought of simply as spring-mass-dashpots. For our setup, suppose we have probability densities $f^{*}(x)$ and $f(x), x \in \Omega=(0,1)$, and interpolating densities $f(x, t), x \in \Omega, 0 \leq t \leq \tau$ with $f^{*}(x)=f(x, 0)$ and $f(x)=f(x, \tau)$. For this 'Eulerian' description, there is a 'Lagrangian' description in terms of a family of measure preserving mappings, transfer functions, homeomorphisms of the interval into itself, $\phi(x, t), x \in \Omega, 0 \leq t \leq \tau$ related by

$$
\int_{\Omega} \zeta(y) f(y, t) d y=\int_{\Omega} \zeta(\phi(x, t)) f^{*}(x) d x .
$$

The velocities in the two descriptions satisfy $\phi_{t}(x, t)=v(\phi(x, t), t)$. For $f(x, t)$ there is

$$
f_{t}+(v f)_{x}=0 \text { in } \Omega, 0<t<\tau \quad \text { (continuity equation) }
$$

and likewise in the 'Lagrangian' version

$$
f(\phi(x, t), t) \phi_{x}=f^{*}(x) .
$$


This is actually the Monge-Ampere Equation. For example, if $v$ is given and we wish to solve (1), (2) corresponds to a characteristic equation.

For the ensemble of spring-mass-dashpots, the viscous dissipation moving from $f^{*}$ to $f$ via $f(x, t)$ is simply

$$
\gamma \int_{0}^{\tau} \int_{\Omega} v^{2} f d x d t
$$

for a parameter $\gamma$. When the system moves in response to a potential $\psi$, its free energy at a density $\varphi$ is

$$
F(\varphi)=\int_{\Omega}(\psi \varphi+\sigma \varphi \log \varphi) d x
$$

In this way, we arrive at a simple mesoscopic dissipation principle. The state $f$ is admissible from $f^{*}$ provided

$$
\gamma \int_{0}^{\tau} \int_{\Omega} v^{2} f d x d t+F(f) \leq F\left(f^{*}\right)
$$

for some interpolating density $f(x, t)$ with $f^{*}(x)=f(x, 0)$ and $f(x)=f(x, \tau)$. We regard $\tau$ as a relaxation time. To connect this to a variational principle, we observe that $[3]$

$$
\frac{1}{2 \tau} d\left(f, f^{*}\right)^{2}=i n f_{A} \frac{1}{2} \int_{0}^{\tau} \int_{\Omega} v^{2} f d x d t
$$

where $A$ is the family of interpolating densities and $d$ is the Kantorovich-Wasserstein metric defined by

$$
\begin{array}{r}
d\left(f, f^{*}\right)^{2}=\inf _{P} \int_{\Omega \times \Omega}|x-y|^{2} d p(x, y) \\
P=\text { joint distributions with marginals } f, f^{*} .
\end{array}
$$

The optimality condition for $f, v$ in (4) is

$$
v_{t}+v v_{x}=0 \text { in } \Omega, 0<t<\tau \quad \text { (Burgers'Equation) }
$$

Its 'Lagrangian' form is the geodesic equation, [3], [16],

$$
\frac{d^{2}}{d t^{2}} d(\phi(x, t), \phi(x, \tau))^{2}=0
$$

which implies

$$
\phi(x, t)=x+\frac{t}{\tau}(\phi(x, \tau)-x), x \in \Omega, 0<t<\tau
$$

The metric $d$ delivers the weak* topology on measures, i.e., its topology as the dual space of $C(\Omega)$, and the 'Lagrangian' form suggests that the optimality condition describes a geodesic path in this space.

For convenience we set $\gamma=\frac{1}{2}$. Our variational principle is now: given $f^{*}$, determine $f$ such that

$$
\frac{1}{2 \tau} d\left(f, f^{*}\right)^{2}+F(f)=\min
$$

The variational principle (5) provides an implicit scheme: Given $f^{(k-1)}$, set $f^{*}=f^{(k-1)}$ and determine $f^{k}$ from the minimum principle. Then define $f^{(\tau)}$

$$
f^{(\tau)}(x, t)=f^{k}(x) \quad k \tau<t \leq(k+1) \tau
$$


The great merit of the Wasserstein metric is that it may be, in essence, differentiated. Thus, in the limit as $\tau \rightarrow 0, f^{(\tau)}$ tends to the solution $f$ of the ordinary Fokker-Planck Equation, [9], [14], [15],

$$
\begin{aligned}
& \frac{\partial f}{\partial t}=\sigma \frac{\partial^{2} f}{\partial x^{2}}+\frac{\partial}{\partial x}\left(\psi^{\prime} f\right) \quad \text { in } \quad \Omega, t>0 \\
& \sigma \frac{\partial}{\partial x} f+\psi^{\prime} f=0 \quad \text { on } \quad \partial \Omega, t>0
\end{aligned}
$$

Variational principles such as (5) above may be considered without discussing natural systems, of course, and there is now a significant literature in this topic, and even traditional problems have unexpected interpretations, [21]. (5) establishes that the coarse graining of the microscopic system gives rise to weak topology dynamics at the mesoscale. For situations, like the one below, where equilibrium is never achieved, this may provide additional insight into their metastable nature.

From the analysis point of view, one observes that the basic variational principle is convex and superlinear, so existence of the iterates in the implicit scheme is not usually a difficulty. Convergence as $\tau \rightarrow 0$ could be, especially for nonlinear problems.

\section{A look at conventional kinesin}

Conventional kinesin has two identical head domains (heavy chains) which walk in a hand over hand fashion along a rigid microtubule. This is an intricate process with a complicated transformation path comprising both the ATP hydrolysis (chemical states) and the motion (mechanical states), [8], [22]. For a crude reckoning, at a gross combinatorial level, each head is attached or in motion and is nucleotide bound or not. Assuming that a given motor has one head bound and one free at any instant leads to eight possible pathways for each cycle. We shall give a simplified description by considering the nucleotide binding and then the subsequent motion. Our dissipation/variational principle is flexible enough to accomodate this process.

The ensemble of motor heads may be divided into two sets, set 1 and set 2 ; for example, the set 1 motors bind to odd labled sites on microtubules and the set 2 motors bind to even labeled sites at a given time $t$.. This permits distance along the microtubule to be used as a process variable. Regard the conformational change and nucleotide binding to be the result of first order chemistry and the motion to be the result of interaction with potentials, diffusion, and dissipation. Let $\rho_{1}$ and

$\rho_{2}$ denote the relative densities of the set 1 and set 2 motors in the powerstroke state. Introduce potentials and coefficients for conformational change,

$$
\begin{aligned}
\sigma & >0 \text { constant } \\
\psi_{i} & \geq 0 \text { and } \nu_{i} \geq 0, i=1,2, \quad \text { smooth and periodic of period } \frac{1}{N}
\end{aligned}
$$


with supp $\nu_{1}=\operatorname{supp} \nu_{2}$ and $\nu_{1}+\nu_{2} \leq 1$. Let

$$
P=\mathbf{1}+\tau\left(\begin{array}{cc}
-\nu_{1} & \nu_{2} \\
\nu_{1} & -\nu_{2}
\end{array}\right)
$$

where $\tau$ is a relaxation time. Denote the free energy of this system by

$$
F(\rho)=\sum_{i=1}^{2} \int_{\Omega}\left(\psi_{i} \rho_{i}+\sigma \rho_{i} \log \rho_{i}\right) d x
$$

We may envision a cycle starting with density $\rho^{*}=\left(\rho_{1}^{*}, \rho_{2}^{*}\right)$ and proceeding by

$$
\rho^{*} \rightarrow \rho^{*} P \rightarrow \rho
$$

subject to the dissipation principle: given $\rho^{*}$ with

$$
\int_{\Omega}\left(\rho_{1}^{*}+\rho_{2}^{*}\right) d x=1 \quad \text { and } \quad \rho_{i}^{*} \geq 0 \text { in } \Omega,
$$

determine $\rho$ by

$$
\begin{aligned}
& \sum_{i=1}^{2} \frac{1}{2 \tau} d\left(\rho_{i},\left(\rho^{*} P\right)_{i}\right)^{2}+F(\rho)=\min \\
& \int_{\Omega} \rho_{i} d x=\int_{\Omega}\left(\rho^{*} P\right)_{i} d x
\end{aligned}
$$

The variational principle (10) separates the roles of the dissipation, conformational change, and free energy in the system. It gives the incremental state of the system in terms of a step in a Markov chain from its prior state. Although there are some subtleties, (10) admits an Euler Equation which is the system [5]

$$
\begin{aligned}
& \frac{\partial \rho_{1}}{\partial t}=\frac{\partial}{\partial x}\left(\sigma \frac{\partial \rho_{1}}{\partial x}+\psi_{1}^{\prime} \rho_{1}\right)-\nu_{1} \rho_{1}+\nu_{2} \rho_{2} \text { in } \Omega, t>0 \\
& \frac{\partial \rho_{2}}{\partial t}=\frac{\partial}{\partial x}\left(\sigma \frac{\partial \rho_{2}}{\partial x}+\psi_{2}^{\prime} \rho_{2}\right)+\nu_{1} \rho_{1}-\nu_{2} \rho_{2} \text { in } \Omega, t>0 \\
& \sigma \frac{\partial \rho_{1}}{\partial x}+\psi_{1}^{\prime} \rho_{1}=0 \text { on } \partial \Omega, t>0 \\
& \sigma \frac{\partial \rho_{2}}{\partial x}+\psi_{2}^{\prime} \rho_{2}=0 \text { on } \partial \Omega, t>0 \\
& \rho_{i}(x, 0)=\rho_{i}^{0} \geq 0, \quad \text { in } \Omega, \quad i=1,2 \\
& \int_{\Omega}\left(\rho_{1}+\rho_{2}\right) d x=1
\end{aligned}
$$

and moreover this system has a solution for all time. The general program to obtain (12), (13) from the variational principle (10) consists of two parts. First there is some type of estimate of iterates and second an approximate Euler Equation. When estimating the left hand side of (5), we choose $f^{*}$ as a test function, which gives

$$
\frac{1}{2 \tau} d\left(f, f^{*}\right)^{2}+F(f) \leq F\left(f^{*}\right)
$$


When applied to the sequence of iterates $\left(f^{k}\right)$, this provides the basic estimate

$$
\begin{gathered}
\frac{1}{2 \tau} \sum_{k=1}^{\infty} d\left(f^{k-1}, f^{k}\right)^{2} \leq F\left(f^{0}\right) \quad \text { and } \\
F\left(f^{k}\right) \leq F\left(f^{0}\right), \quad k=1,2,3, \ldots
\end{gathered}
$$

In our variational principle $(10), \rho^{*} P$ is an admissible competitor but $\rho^{*}$ is not. Hence

$$
\sum_{i=1}^{2} \frac{1}{2 \tau} d\left(\rho_{i},\left(\rho^{*} P\right)_{i}\right)^{2}+F(\rho) \leq F\left(\rho^{*} P\right)
$$

To replace $\rho^{*} P$ by $\rho^{*}$ in (14), we use the simple property of Markov chains that relative entropy of successive states decreases. Namely, for a probablity matrix $P$ with stationary state $\mu^{\sharp}$, given a vector of non-negative components $\mu$,

$$
\sum_{j=1}^{n}(\mu P)_{j} \log \frac{(\mu P)_{j}}{\mu_{j}^{\sharp}} \leq \sum_{j=1}^{n} \mu_{j} \log \frac{\mu_{j}}{\mu_{j}^{\#}}
$$

For the 2 matrix $P$, the (x-dependent) stationary state is just proportional to $\left(\nu_{2}, \nu_{1}\right)$ so we obtain

$$
\sum_{i=1}^{2} \frac{1}{2 \tau} d\left(\rho_{i},\left(\rho^{*} P\right)_{i}\right)^{2}+F(\rho) \leq F\left(\rho^{*}\right)+\text { const. } \tau
$$

This estimate is sufficient to establish the approximate Euler equation

$$
\begin{aligned}
& \left|\sum_{i=1,2} \int_{\Omega}\left\{\left(\frac{1}{\tau}\left(\rho_{i}-\rho_{i}^{*}\right)-\left(\rho^{*} \nu\right)_{i}\right) \zeta_{i}-\sigma \rho_{i} \zeta_{i}^{\prime \prime}+\psi_{i}^{\prime} \rho_{i} \zeta_{i}^{\prime}\right\} d x\right| \\
& \leqq \frac{1}{2} \max \sup \left|\zeta_{i}^{\prime \prime}\right|\left(F\left(\rho^{*}\right)-F(\rho)+C \tau\right), \quad \zeta \in C_{0}^{\infty}(\Omega)
\end{aligned}
$$

and to prove that the sequence $\left.\rho^{(} \tau\right)$,

$$
\rho^{(\tau)}(x, t)=\rho^{k}(x) \quad k \tau<t \leq(k+1) \tau,
$$

converges as $\tau \rightarrow 0$ to a solution of (12), (13). Along the way, we are assisted by a novel maximum principle. Suppose that $\rho$ is the solution of the (10) for $\rho^{*}$. If

$$
\frac{\rho_{i}^{*}}{e^{-\psi_{i} / \sigma}} \leq M_{i}
$$

then

$$
\frac{\rho_{i}}{e^{-\psi_{i} / \sigma}} \leq M_{i}(1+\alpha \tau) \quad i=1,2
$$

for a suitable $\alpha>0$. The interesting feature is that the proof is a truncation argument involving joint distributions. The first use of the idea was by Otto, [15], and new ingredients have been added to it by Petrelli and Tudorascu, [19]. There is a similar minimum principle. These estimates do not permit us to deduce the behaviour of the system as $t \rightarrow \infty$, which will be dealt with elsewhere.

The foregoing may be generalized easily to $n$ species with potentials $\psi_{i}$ and a matrix $\nu=\left(\nu_{i j}\right)$, with $\nu_{i j}>0$ for $i \neq j$ and $\sum_{j=1}^{n} \nu_{i j}=0$. i.e., $P$ as defined above a probability matrix. Allowing more complex interactions among the species requires more thought about the form of the interactions and their statistical properties. 


\section{The stationary solution}

There is, in addition, a unique stationary solution $\rho^{\sharp}$ of $(12)$, (13) provided

$$
\nu_{1} \geq 0 \text { and } \nu_{2} \geq 0
$$

and neither are identically zero. Namely, $\rho^{\sharp}$ is the solution of the system of ordinary differential equations [4]

$$
\begin{aligned}
& \frac{d}{d x}\left(\sigma \frac{d \rho_{1}^{\sharp}}{d x}+\psi_{1}^{\prime} \rho_{1}^{\sharp}\right)-\nu_{1} \rho_{1}^{\sharp}+\nu_{2} \rho_{2}^{\sharp}=0 \text { in } \Omega \\
& \frac{d}{d x}\left(\sigma \frac{d \rho_{2}^{\sharp}}{d x}+\psi_{2}^{\prime} \rho_{2}^{\sharp}\right)+\nu_{1} \rho_{1}^{\sharp}-\nu_{2} \rho_{2}^{\sharp}=0 \text { in } \Omega \\
& \sigma \frac{d \rho_{1}^{\sharp}}{d x}+\psi_{1}^{\prime} \rho_{1}^{\sharp}=0 \text { on } \partial \Omega \\
& \sigma \frac{d \rho_{2}^{\sharp}}{d x}+\psi_{2}^{\prime} \rho_{2}^{\sharp}=0 \text { on } \partial \Omega \\
& \int_{\Omega}\left(\rho_{1}^{\sharp}+\rho_{2}^{\sharp}\right) d x=1
\end{aligned}
$$

Note that in general $\rho^{\sharp}$ does not minimize (8). There are two ways to attack this, one starting with the Schauder Fixed Point Theorem and one by a shooting method, based on writing (17),(18) as a first order system, [4].
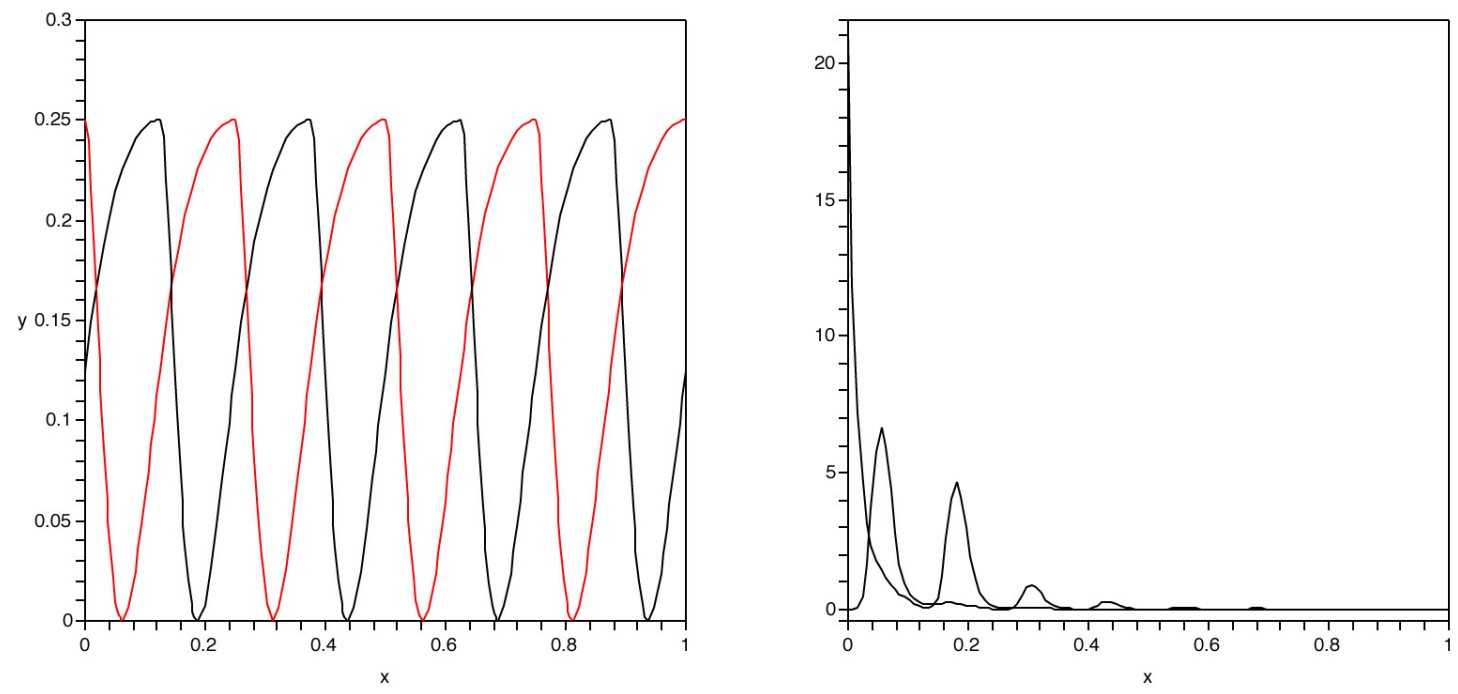

Figure 1: Interdigitated asymmetric potentials $\psi_{1}$ and $\psi_{2}$ (left) and stationary state $\rho^{\sharp}$ demonstrating about 0.9 of its mass on the left half of the interval.

We would like to briefly discuss the origins of transport and the role of the asymmetry of the potentials. Assume that $\psi_{1}$ and $\psi_{2}$ are periodic of period $1 / N$, in fact, for purposes of discussion, 


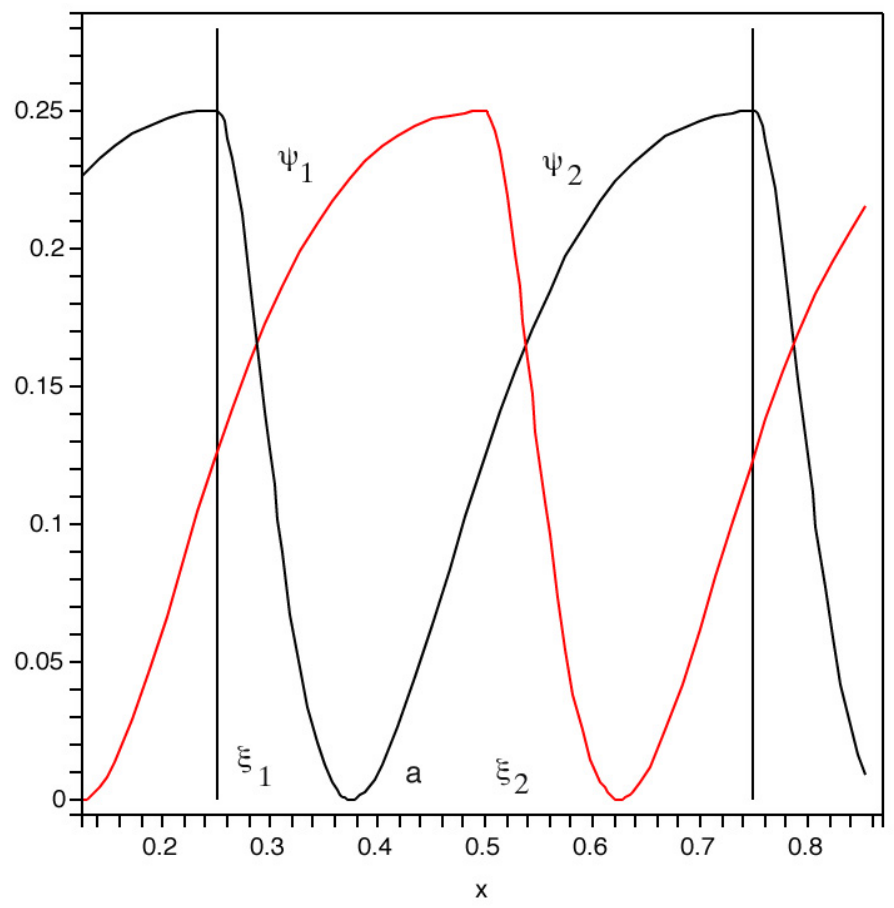

Figure 2: For this pair of $\psi_{1}$ and $\psi_{2}$, there is no interval where both are decreasing and transport to the left is anticipated

let us take

$$
\psi_{2}(x)=\psi_{1}\left(x-\frac{1}{2 N}\right)
$$

so that they interdigitate each other. Assume that $\psi_{i}$ decreases monotonely from its maximum to its minimum and then increases monotonely to its maximum in each period interval. Choose a period interval, max to max, for $\psi_{1}$, say $\left[\xi_{1}, \xi_{1}+1 / N\right]$ and suppose we are in the situation where

$$
\begin{gathered}
\xi_{1}<a<\xi_{2}<b<\xi_{1}+1 / N \text { and } \\
\psi_{1}\left(\xi_{1}\right)=\psi_{1}\left(\xi_{1}+1 / N\right)=\max \psi_{1}, \quad \psi_{1}(a)=\min \psi_{1}=0 \\
\psi_{2}\left(\xi_{2}\right)=\max \psi_{2}, \quad \psi_{2}(b)=\min \psi_{2}=0
\end{gathered}
$$

Think of $\sigma$ as very small. Now we have that

1. in $\left(a, \xi_{2}\right), \psi_{1}^{\prime}>0$ and $\psi_{2}^{\prime}>0$, so $\rho_{1}^{\sharp}$ and $\rho_{2}^{\sharp}$ are both exponentially decreasing regardless of $\nu_{i}$.

2. in $\left(\xi_{1}, a\right)$, there is a large population of $\rho_{1}^{\sharp}$, and, because of the equations (17), (18), some is passed to $\rho_{2}^{\sharp}$ because $\nu_{2}>0$. Little is passed from $\rho_{2}^{\sharp}$ to $\rho_{1}^{\sharp}$ because we are not close to the minimum of $\psi_{2}$.

3. the net effect is movement to the left 
The condition for the balance in 2 , and for similar behavior near the minima of $\psi_{2}$, is that

- $\psi_{1}$ is increasing where $\psi_{2}$ is decreasing and $\psi_{2}$ is increasing where $\psi_{1}$ is decreasing.

This means, in particular, that the minima of the $\psi_{i}^{\prime} s$ are located asymmetrically in their period intervals. Unfortunately, the above reads like just one of many plausible scenarios and so does not serve well for intuition, but it is the correct one. The result may be loosely formulated in this way:

Suppose there is no interval where $\psi_{1}$ and $\psi_{2}$ are both decreasing, and

$$
\nu_{1}>0 \text { and } \nu_{2}>0 \text { in } \Omega
$$

then

$$
\rho_{1}^{\sharp}\left(x+\frac{1}{N}\right)+\rho_{2}^{\sharp}\left(x+\frac{1}{N}\right) \leq K e^{-\frac{C}{\sigma}}\left(\rho_{1}^{\sharp}(x)+\rho_{2}^{\sharp}(x)\right), \quad x \geq 1+\frac{2}{N}
$$

To prove this result, we rewrite (12), (13) as the first order system (dropping the ${ }^{\sharp}$ superscript), with

$$
\begin{gathered}
\phi=\sigma \rho_{1}^{\prime}+\psi_{1}^{\prime} \rho_{1}, \\
\sigma \rho_{1}^{\prime}=\phi-\psi_{1}^{\prime} \rho_{1} \\
\sigma \rho_{2}^{\prime}=-\phi-\psi_{2}^{\prime} \rho_{2} \\
\phi^{\prime}=\nu_{1} \rho_{1}-\nu_{2} \rho_{2} \\
\phi(0)=\phi(1)=0
\end{gathered}
$$

Or

$$
\rho^{\prime}=A \rho, \text { with } \rho=\left(\begin{array}{c}
\rho_{1} \\
\rho_{2} \\
\phi
\end{array}\right) \quad \text { and } A=\frac{1}{\sigma}\left(\begin{array}{ccc}
-\psi_{1}^{\prime} & 0 & 1 \\
0 & -\psi_{2}^{\prime} & -1 \\
\sigma \nu_{1} & -\sigma \nu_{2} & 0
\end{array}\right)
$$

Let $R(\xi, x)$ be a fundamental solution to this system with $R(\xi, \xi)=1$, say. Write

$$
R=\left(\begin{array}{ccc}
\rho_{11} & \rho_{12} & \rho_{13} \\
\rho_{21} & \rho_{22} & \rho_{23} \\
\phi_{1} & \phi_{2} & \phi_{3}
\end{array}\right)
$$

Thus, in particular,

$$
\rho(a)=R\left(\xi_{1}, a\right) \rho\left(\xi_{1}\right) \quad \text { and } \quad \rho\left(\xi_{2}\right)=R\left(a, \xi_{2}\right) \rho(a)
$$

Since $\rho_{i}>0$, the additional function $\phi$ can be eliminated from the equation in favor of an inequality. Indeed,

$$
\begin{array}{ll}
0<\rho_{1}(x)=\rho_{11} \rho_{1}(\xi)+\rho_{12} \rho_{2}(\xi)+\rho_{13} \phi(\xi), & x<\xi \\
0<\rho_{2}(x)=\rho_{21} \rho_{1}(\xi)+\rho_{22} \rho_{2}(\xi)+\rho_{23} \phi(\xi), & x<\xi
\end{array}
$$


where the $\rho_{i j}$ are evaluated at $x$. Hence,

$$
\begin{aligned}
& \phi(\xi)<-\frac{\rho_{11}}{\rho_{13}} \rho_{1}(\xi)-\frac{\rho_{12}}{\rho_{13}} \rho_{2}(\xi) \text { and } \\
& \phi(\xi)<-\frac{\rho_{21}}{\rho_{23}} \rho_{1}(\xi)-\frac{\rho_{22}}{\rho_{23}} \rho_{2}(\xi)
\end{aligned}
$$

Combining this with (25), (26) and reconfiguring gives that

$$
\begin{aligned}
& \rho_{1}(x)<\frac{\rho_{13} \rho_{21}-\rho_{11} \rho_{23}}{-\rho_{23}} \rho_{1}(\xi)+\frac{\rho_{22} \rho_{13}-\rho_{12} \rho_{23}}{-\rho_{23}} \rho_{2}(\xi) \\
& \rho_{2}(x)<\frac{\rho_{13} \rho_{21}-\rho_{11} \rho_{23}}{\rho_{13}} \rho_{1}(\xi)+\frac{\rho_{22} \rho_{13}-\rho_{12} \rho_{23}}{\rho_{13}} \rho_{2}(\xi)
\end{aligned}
$$

A first thought is that when a typical $\rho_{i j}$ varies with $\exp (c / \sigma)$, the fraction varies like $\exp (c / \sigma)^{2} / \exp (c / \sigma)=\exp (c / \sigma)$, that is, exponential in $1 / \sigma$. Interesting here is that the numerators in the fractions are the terms $(\operatorname{adj} R)_{23}$ and $(\operatorname{adj} R)_{13}$ and the adjugate itself satisfies an equation (variation of Abel's formula)

$$
\frac{d}{d x} \operatorname{adj} R=\operatorname{adj} R M, \quad M=(\operatorname{trace} A) \mathbf{1}-A
$$

which means that the numerator and the denominator are typically of the same order. This is the starting point of the proof. The details require careful analysis of $R$ and $a d j R$ in the appropriate intervals.

With Bryce McLeod, we are preparing a second approach which would extend to an arbitrary number of components $\rho_{i}$ weakly coupled by matrix $N=\left(\nu_{i j}\right)$.

At this writing, the relationship of the supports of the conformational change coefficients $\nu_{i}$ and the potentials $\psi_{i}$ is still not clear. One obvious situation where no transport can be expected is when the system (12), (13) decouples. This happens when

$$
\nu \propto\left(e^{-\frac{\psi_{2}}{\sigma}}, e^{-\frac{\psi_{1}}{\sigma}}\right)
$$

This is sometimes referred to as detailed balance, but it only concerns the balance in part of the equations. However, even in this case, retaining the $\sigma=\sigma_{0}$ above in (27) but diminishing sufficiently the diffusion coefficient $\sigma$ in (12), (13) will result in transport according to our theorem provided the $\nu_{i}$ are positive.

A more amazing result is given in the last figure. Here the potentials are the same as before, although there are eight periods instead of four, but the support of the $\nu_{i}$ are where one $\psi_{j}$ is decreasing and the other increasing. The result is transport in the reverse, that is the "wrong" direction. Much remains to be studied in these problems.

\section{References}

[1] Adjari, A. And Prost, J. (1992) Mouvement induit par un potentiel périodique de basse symétrie: dielectrophorese pulse, C. R. Acad. Sci. Paris t. 315, Série II, 1653. 


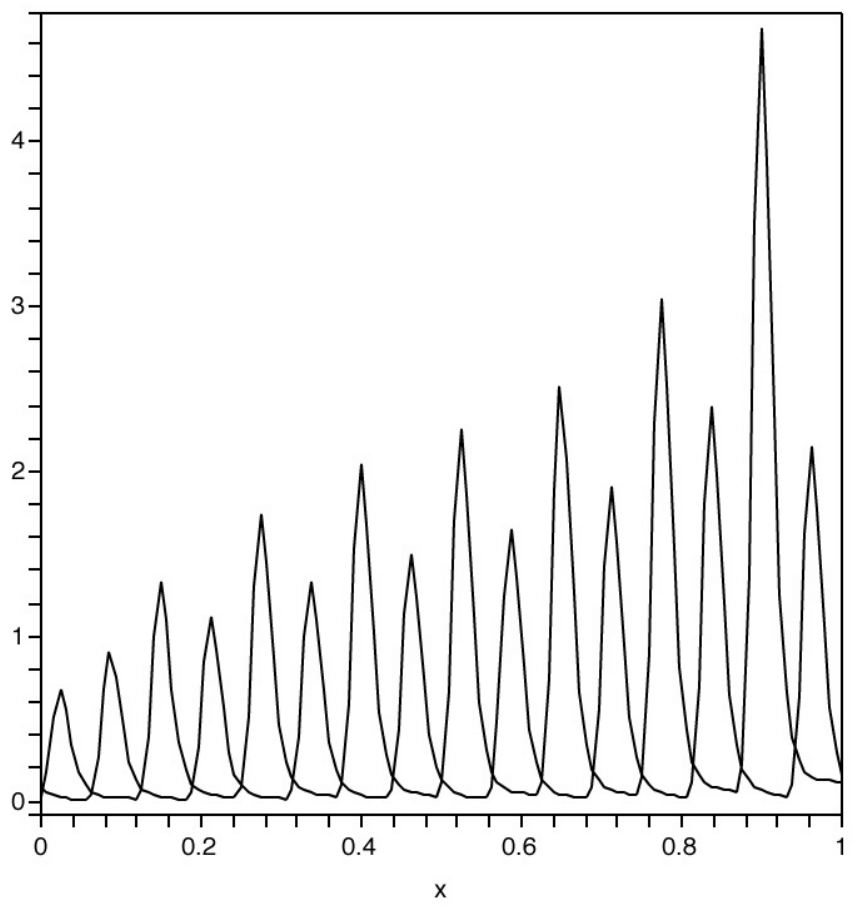

Figure 3: Reverse transport achieved by selecting the support of the $\nu_{i}$ in a region where one $\psi_{i}$ is increasing and the other decreasing

[2] Astumian, R.D. (1997) Thermodynamics and kinetics of a Brownian motor, Science 276 (1997), 917-922.

[3] Benamou, J.-D. And Brenier, Y. (2000) A computational fluid mechanics solution to the Monge-Kantorovich mass transfer problem, Numer. Math. 84, 375-393.

[4] Chipot, M., Hastings, S., And Kinderlehrer, D., to appear

[5] Chipot, M., D. Kinderlehrer, D. And Kowalczyk, M. (2003) A variational principle for molecular motors, Meccanica, 38, 505-518

[6] Dolbeault, J., Kinderlehrer, D., And Kowalczyk, M. Remarks about the flashing rachet, to appear Proc. PASI 2003

[7] Heath, D., Kinderlehrer, D. And KowalczyK, M. (2002) Discrete and continuous ratchets: from coin toss to molecular motor, Discrete and continuous dynamical systems Ser. B 2 no. $2,153-167$.

[8] Howard, J. (2001) Mechanics of Motor Proteins and the Cytoskeleton, Sinauer Associates, Inc., 2001.

[9] Jordan, R., Kinderlehrer, D. and Otto, F. (1998) The variational formulation of the Fokker-Planck equation, SIAM J. Math. Anal. Vol. 29 no. 1, 1-17. 
[10] Kinderlehrer, D. And Kowalczyk, M (2002) Diffusion-mediated transport and the flashing ratchet, Arch. Rat. Mech. Anal. 161, 149-179.

[11] Kinderlehrer, D. And Walkington, N. (1999) Approximation of parabolic equations based upon Wasserstein's variational principle, Math. Model. Numer. Anal. (M2AN) 33 no. 4, $837-852$.

[12] Okada, Y. And Hirokawa, N. (1999) A processive single-headed motor: kinesin superfamily protein KIF1A, Science Vol. 283, 19

[13] Okada, Y. and Hirokawa, N. (2000) Mechanism of the single headed processivity: diffusional anchoring between the K-loop of kinesin and the $\mathrm{C}$ terminus of tubulin, Proc. Nat. Acad. Sciences 7 no. 2, 640-645.

[14] Отто, F. (1998) Dynamics of labyrinthine pattern formation: a mean field theory, Arch. Rat. Mech. Anal. 141, 63-103

[15] Отто, F. (2001) The geometry of dissipative evolution equations: the porous medium equation, Comm. PDE 26, 101-174

[16] Otto, F. And Villani, C. (2000) Generalization of an inequality by Talagrand and links with the logarithmic Sobolev Inequality, J. Funct. Anal. 173, 361-400

[17] Parmeggiani,A., Jülicher, F., Adjari, A. And Prost, J. (1999) Energy transduction of isothermal ratchets: generic aspects and specific examples close and far from equilibrium, Phys. Rev. E, 60 no. 2, 2127-2140.

[18] Peskin, C.S.. Ermentrout, G.B. And Oster, G.F. (1995) The correlation ratchet: a novel mechanism for generating directed motion by ATP hydrolysis, in Cell Mechanics and Cellular Engineering (V.C Mow et.al eds.), Springer, New York

[19] Petrelli, L. and Tudorascu, A. Variational principle for general Fokker-Planck equations , to appear

[20] Reimann, P. (2002) Brownian motors: noisy transport far from equilibrium, Phys. Rep. 361 nos. 2-4, 57-265.

[21] Tudorascu, A. A one phase Stefan problem via Monge-Kantorovich theory,( CNA Report 03-CNA-007)

[22] Vale, R.D. And Milligan, R.A. (2000) The way things move: looking under the hood of motor proteins, Science 288, 88-95.

[23] C. Villani (2003) Topics in optimal transportation, AMS Graduate Studies in Mathematics vol. 58, Providence 